\title{
Whole body vibration to attenuate reduction of explosive force in chronic kidney disease patients: a randomized controlled trial
}

\author{
Helen K. B. Fuzari', Armèle Dornelas de Andrade'1, Mikhail Santos Cerqueira² ${ }^{2}$ Rafael Pereira², Ana I. C. Medeiros' ${ }^{1}$, Jéssica C. Leite', \\ Elaine C. S. C. Moura', Helga C. M. Souza', Claudia Regina O. P. Lima³, Patrícia Érika de Melo Marinho1,* \\ 'Physiotherapy Department, Cardiopulmonary Physicaltherapy Laboratory, Universidade Federal de Pernambuco, Recife, Brazil \\ ${ }^{2}$ Biological Sciences Department, Neuromuscular Physiology Research Group, Universidade Estadual do Sudoeste da Bahia, Jequié, Bahia, Brazil \\ ${ }^{3}$ Statistics Department, Unversidade Federal de Pernambuco, Recife, Brazil
}

To investigate whether whole body vibration (WBV) training increases the explosive force of the knee extensors in chronic kidney disease (CKD) patients. Fourteen CKD patients undergoing hemodialysis were randomly allocated in WBV training or Sham group. Explosive force parameters (contractile impulse [CImp] and relative rate of force development [RFDr]) obtained in early ( 30 and $50 \mathrm{msec}$ ) and late phases (100 and $200 \mathrm{msec}$ ) of the knee extensors force/time curve. CImp and RFDr obtained at the early phase of force/time curve reduced after the intervention period, with a smaller decline for WBV (CImp at 50 msec [ - $15 \%$ and $-51 \%$, $P=0.038]$, RFDr at $30 \mathrm{msec}[\sim-22 \%$ and $-52 \%, P=0.044]$ and RFDr at 50 msec $[\sim-11 \%$ and $-54 \% ; P=0.008])$. In the late phase there was a lower decline for WBV group compared to Sham group, respectively: CImp: $100 \mathrm{msec}(\sim-8 \%$ and $-55 \%, P=0.025), 200 \mathrm{msec}(\sim-3 \%$ and $-46 \%, P=$ $0.025)$; RFDr $100 \mathrm{msec}(\sim 0.01 \%$ and $-56 \%, P=0.033), 200 \mathrm{msec}(\sim-5 \%$ and $-36 \%, P=0.004)$. Three months of WBV training may attenuate the explosive force reduction in CKD patients.

Keywords: Rate of force development, Contractile impulse, Muscle weakness, Hemodialysis

\section{INTRODUCTION}

Chronic kidney disease (CKD) affects many organs and systems, leading to exercise intolerance due to the musculoskeletal system impairments. As a consequence, physical inactivity, functional capacity and quality of life declines are commonly reported in this population (Adams and Vaziri, 2006; Bogaerts et al., 2009; Kopple et al., 2005).

Muscle weakness, especially involving lower limbs is a consequence of persistent uremia from CKD patients, which is aggravated by a sedentary lifestyle (Cheema et al., 2007). On the other hand, training programs with endurance or strength exercises have been proposed to CKD patients undergoing hemodialysis (HD) (Chen et al., 2010; Storer et al., 2005). However, in clinical practice, some training protocols are difficult to be supported by CKD patients, owing to the exercise intensity and/or patient limitations, as the clinical condition or the presence of comorbidities.

Whole body vibration (WBV) is an alternative method for strength and neuromuscular training (Perchthaler et al., 2015). Improvements in neuromuscular performances with WBV training are related to tonic vibration reflex (TVR), which involve muscle spindles and fast-adapting mechanoreceptors (Rittweger, 2010). WBV has shown promising results in muscle performance for elderly (Bogaerts et al., 2007a) and for a wide range of patients, as Parkinson disease patients (Haas et al., 2006), multiple sclerosis (Schuhfried et al., 2005), stroke (Pang et al., 2013), cystic fibrosis (Rietschel et al., 2008), and chronic obstructive pulmonary disease patients (Greulich et al., 2014).
*Corresponding author: Patrícia Érika de Melo Marinho

(iD) https://orcid.org/0000-0002-3093-7481

Department of Physical Therapy, Universidade Federal de Pernambuco - UFPE, Recife, Av. Jorn. Anibal Fernandes, s/n, Cidade Universitária CEP: 50740-560, Brazil Tel: +55-81-2126-8490, Fax: +55-81-2126-8491, E-mail: patmarinho@yahoo.com.br Received: May 28, 2018 / Accepted: August 8, 2018
This is an Open Access article distributed under the terms of the Creative Commons Attribution Non-Commercial License (http://creativecommons.org/licenses/by-nc/4.0/) which permits unrestricted non-commercial use, distribution, and reproduction in any medium, provided the original work is properly cited. 
Furthermore, WBV training has emerged as an interesting alternative to reduce risk of fall in older adults (Lam et al., 2012). In fact, a recent study showed a significant reduction of falls risk from elders submitted to 8 weeks of WBV training (Yang et al., 2015). Interestingly, CKD patients undergoing HD have a high risk of falling, leading to suggestions regarding the prevention strategies to this population (Cook et al., 2006) and the WBV training could be proposed as an promising intervention.

In daily life activities, many motor responses, such as the prevention of falls, are characterized by a rapid force development (0$200 \mathrm{msec}$ ). This reaction time is considerably shorter than the time required (between 400-600 msec) to reach the peak or maximum force (Aagaard et al., 2002). In this context, the ability to develop force at the beginning of a muscle contraction, also known as explosive force, may be functionally more important than peak force (Schettino et al., 2014).

Notwithstanding, it was identified that explosive force decline earlier (Schettino et al., 2014) and is better correlated with functional mobility, than the peak force in healthy elders (Borges et al., 2015). Additionally, compared to the peak force, explosive force has shown a better ability to predict falls among elders (Skelton et al., 2002). Thus, use of strategies focused to increase the explosive force may be important in patients with CKD.

Some studies have demonstrated increase in explosive force after an exercise training period for elders (Sundstrup et al., 2016; Wang et al., 2017), however no studies were found evaluating the effects of WBV training on explosive force in CDK patients.

Therefore, the aim of this study was to investigate whether three months of WBV training increases the explosive force in CKD patients undergoing HD.

\section{MATERIALS AND METHODS}

A randomized controlled pilot trial with blinding of evaluators, participants and statistician was conducted. The study protocol was approved by the institutional committee and the protocol (CAAE: 35872014.8.0000.5208) complied with the Declaration of Helsinki and informed consent was obtained from all participants (Clinical Trials No. NCT02413073).

After randomization in online software (randomization.com), all eligible participants were allocated to one of the groups: WBV or sham. The confidentiality (allocation concealment) was done using black envelopes, opaque, sealed and numbered, prepared by a researcher not involved in the study. Both patients who were in the WBV group and sham group received the same training pro- tocol on the vibrating platform. In the sham group the platform was connected with the display lit, but without vibration, only with the engine noise that reproduced the sound of vibration. The explosive force obtained from a maximum isometric voluntary contraction (MIVC) was considered as an outcome measure.

\section{Subjects}

During the period available for collection (2 years), fourteen CKD patients voluntarily (consecutive sample) participated in the study according to the inclusion criteria: hemodynamically stable (systolic blood pressure $\leq 140 \mathrm{mmHg}$, diastolic blood pressure $\leq 90 \mathrm{mmHg}$, and heart rate $\leq 80 \mathrm{bpm}$ ), in stage 5 or undergoing HD treatment for at least 3 months and without the musculoskeletal impairments that hamper the performance of tests and/or training. Initially, baseline characteristics of sociodemographic, anthropometric and laboratory data from selected patients were recorded (Table 1). The groups were comparable for all variables, except for creatinine values.

Patients with pacemaker, screws and/or pins in the body, acute migraine episodes, cognitive deficits, visual and/or hearing impairment that could limit the evaluation procedures and/or training were excluded; patients classified as physically active according to the International Physical Activity Questionnaire, history of heart surgery, higher body weight than supported by the used

Table 1. Baseline anthropometric and laboratory characteristics of patients in the WBV and sham groups

\begin{tabular}{lccc}
\hline Variable & WBV $(\mathrm{n}=7)$ & Sham $(\mathrm{n}=7)$ & P-value \\
\hline Sex, male:female (\%) & $85.7: 14.3$ & $57.1: 42.9$ & \\
Age (yr) & $62.42 \pm 10.32$ & $53.28 \pm 8.26$ & 0.092 \\
Weight (kg) & $71.94 \pm 8.85$ & $73.80 \pm 15.99$ & 0.793 \\
Height (m) & $1.61 \pm 0.07$ & $1.59 \pm 0.08$ & 0.656 \\
BMl (kg/m²) & $27.75 \pm 3.61$ & $28.83 \pm 5.38$ & 0.667 \\
Time of HD (mo) & $41.86 \pm 29.70$ & $98.14 \pm 96.66$ & 0.167 \\
Laboratory parameter & & & \\
Urea (mg/dL) & $145.71 \pm 35.57$ & $152.42 \pm 27.33$ & 0.699 \\
Creatinine (mg/dL) & $9.14 \pm 2.89$ & $13.88 \pm 2.70$ & $0.009^{*}$ \\
Calcium (mg/dL) & $8.84 \pm 0.77$ & $8.77 \pm 0.28$ & 0.822 \\
Phosphorus (mL) & $5.31 \pm 1.81$ & $6.24 \pm 2.22$ & 0.408 \\
Sodium (mEq) & $134.14 \pm 5.30$ & $137.57 \pm 4.42$ & 0.214 \\
Potassium (mEq) & $4.75 \pm 1.15$ & $4.84 \pm 0.65$ & 0.869 \\
Hemoglobin (g/100 mL) & $11.76 \pm 1.07$ & $12.05 \pm 1.67$ & 0.707 \\
Hematocrit (\%) & $35.72 \pm 2.59$ & $36.90 \pm 4.65$ & 0.572 \\
Albumin (g/dL) & $3.91 \pm 0.21$ & $4.02 \pm 0.28$ & 0.413 \\
\hline
\end{tabular}

Values are presented as mean \pm standard deviation unless otherwise indicated. WBV, whole body vibration; BMI, body mass index; $\mathrm{HD}$, hemodialysis. ${ }^{*} P<0.05$. 


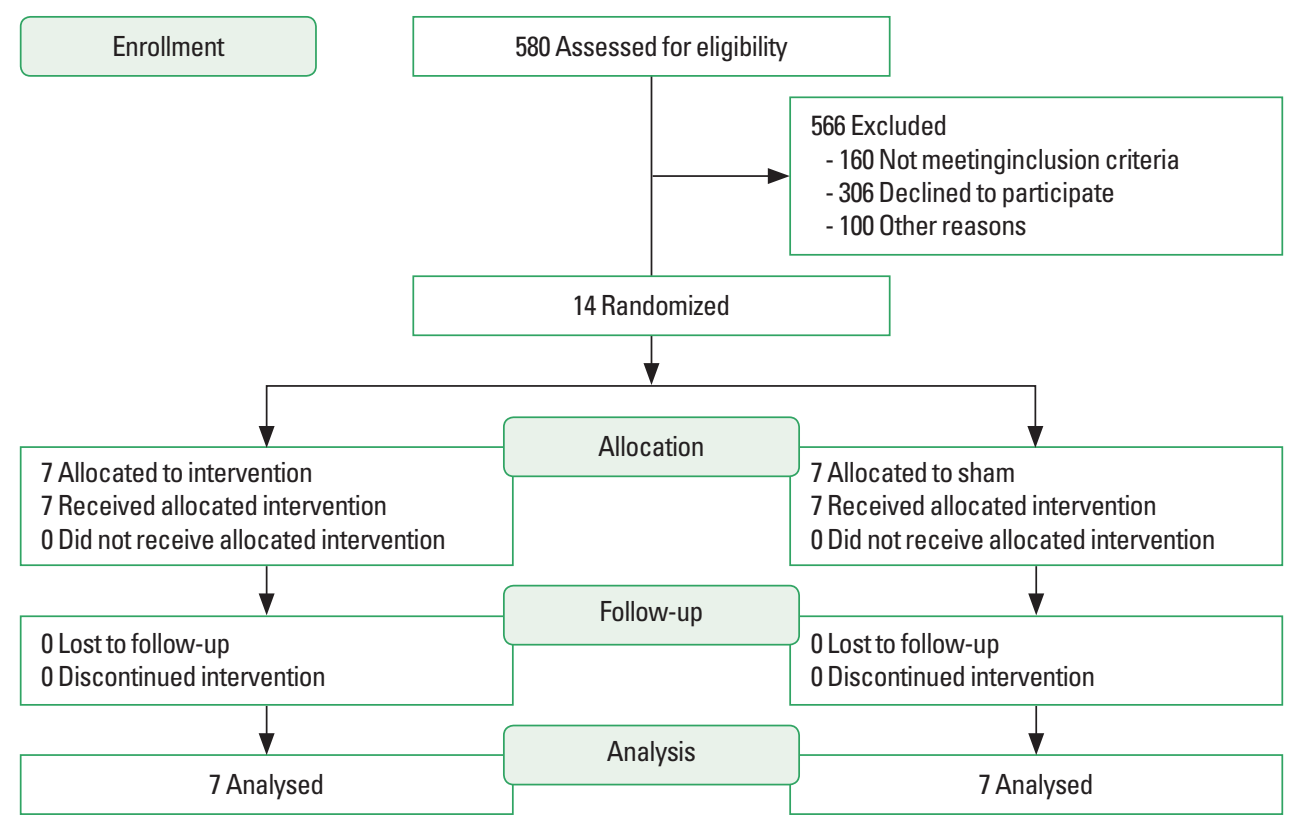

Fig. 1. Flowchart which describes the recruitment, randomization and allocation of patients.

vibratory platform (over $120 \mathrm{~kg}$ ), and using any medication that were not the usual for HD and common comorbidities (diabetes mellitus, hypertension) were also excluded. The study was conducted from April 2015 to February 2016. Fig. 1 shows the flow of participants in the study.

\section{Peak force and explosive force recording and analysis}

It was performed by a blinded evaluator on the dominant limb and in a sitting position, with hip and knee positioned at $90^{\circ}$ of flexion. To choose the dominant limb, the individual told with which leg he/she kicks better. An anklet was adapted to the patient and was associated with a strain gauge (Model 830C, EMG System, São Paulo, Brazil) with capacity for $2000 \mathrm{~N}$ connected to an acquisition system (EMG System). Recordings were sampled at $2 \mathrm{kHz}$ and subjects were carefully instructed to perform a isometric knee extension "as fast and forcefully as possible" after the command "go," sustaining the contraction for 5 to $7 \mathrm{sec}$, when the command "stop" was given.

Each volunteer carried out three MIVC with 1-min rest interval between them. For registration purposes, we adopted the average of three movements that did not exceed $10 \%$ difference among them. Strength data were collected before and three months after training.

The strain gauge signal was smoothed by a digital fourth-order, zero-lag Butterworth filter, with a cutoff frequency of $15 \mathrm{~Hz}$ (Aagaard et al., 2002). Contractile impulse (CImp) and rate of force development (RFD) were obtained as the explosive force parameters, since they are complementary ways to measure the capacity to increase force in short-time periods (Schettino et al., 2014). Clam was obtained as the cumulated area under the forcetime curve, which reflects the entire time history of the contraction. The RFD was obtained as the average slope of the force-time curve ( $\Delta$ force/ $\Delta$ time) over the time intervals of $0-30,0-50$, $0-100$, and $0-200$ msec relative to the onset of contraction (Aagaard et al., 2002).

Onset of muscle contraction was defined as the time point at which the force curve exceeded the baseline by $2.5 \%$ of the difference between baseline force and the maximum voluntary contraction (i.e., maximum extension knee force) (Aagaard et al., 2002). RFD was normalized (relative RFD [RFDr]) by MIVC (\% MIVC/ sec) and calculated at the same time epochs used for RFD (Oliveira et al., 2013). All analyses were conducted using specific algorithms. Table 2 presents the baseline (i.e., data obtained previously to the training or sham period) values and post WBV training values for explosive force parameters.

\section{Intervention}

Participants received individual training in a ventilated and airconditioned room, with sessions lasting about one hour per patient. The WBV training was developed on a vibrating platform (Power Plate MY3, London, UK) with vertical vibration, $35 \mathrm{~Hz}$ fixed frequency and amplitude of $2 \mathrm{~mm}$ and/or $4 \mathrm{~mm}$. The protocol was 
implemented during a 3-month period with two sessions per week, and on alternate days to patients' HD. The range was $2 \mathrm{~mm}$ in the first 2 weeks, and $4 \mathrm{~mm}$ in the following days, and the vibration time increased from $10 \mathrm{~min}$ to $15 \mathrm{~min}$ to $20 \mathrm{~min}$ in the first, second and third months, respectively.

The WBV session consisted of 1-min vibration bouts, interspersed with $30 \mathrm{sec}$ of passive rest (Storer et al., 2005). The patient stood on the platform and remained in a semisquat static position (knees at $30^{\circ}$ flexion; measured with goniometer) during vibration period, and the upper limb contralateral to the arteriovenous fistula, slightly flexed while supporting on the platform and with feet $20 \mathrm{~cm}$ apart (Braz Júnior et al., 2015). During rest the patients were able to extend the knees to relax.

Before each training session the patient performed active stretching of scalene, sternocleidomastoid, hamstring and quadriceps. Blood pressure, heart rate, peripheral oxygen saturation and the perceived effort were monitored at every 5 min during the rest period of training session. Blood pressure was recorded using a sphygmomanometer and a stethoscope (Premium, Rio de Janeiro, Brazil), heart rate and the peripheral oxygen saturation with a pulse oximeter (Ônix 9500, Nonin Medical Inc., Plymouth, MN, USA) and perceived effort with the Borg scale.

For the Sham group, the platform was modified with an externally motor coupled at its base. This apparatus was developed to reproduce the sound of the connected platform. The vibration generated by this apparatus is limited to the external motor and its protective case, being not sufficient to produce any physiological effect (Pereira and Neves, 2006), allowing to blind the patients regarding to the applied intervention throughout the training.

\section{Statistical analysis}

The Shapiro-Wilk test was used to determine whether the variables were normally distributed. For the variables Clam and RFDr at 100 and $200 \mathrm{msec}$ which were not normally distributed, the data are in median \pm interquartile range and Mann-Whitney was used to between group's comparisons. The variables Clam and RFDr at 30 and 50 msec are in mean \pm standard error and Student $t$-test was used to between group's comparisons. Data obtained after the intervention period were normalized by the baseline measures to obtain percent changes of each group and allowing group comparisons independently of baseline discrepancies.

A significance level of $P<0.05$ was used for all statistical procedures, and all statistical analyses were performed using IBM SPSS Statistics ver. 21.0 (IBM Co., Armonk, NY, USA).

\section{RESULTS}

Baseline characteristics of sociodemographic, anthropometric and laboratory data from selected patients are presented in Table 1. The absolute values (i.e., nonnormalized values) of the explosive force parameters (CImp and RFDr) did not exhibit significant differences between groups before and after training (Table 2).

The normalized CImp and RFDr reduced significantly less in CKD patients submitted to 3 months of WBV training when compared to sham group (Figs. 2, 3). Normalized explosive force parameters obtained at the early phase of force/time curve reduced after the intervention period, with a significantly greater decline for the CKD patients submitted to the sham intervention for the CImp at $50 \mathrm{msec}$ and RFDr at 30 and 50 msec: CImp: $30 \mathrm{msec}(\sim-22 \%$ and $-49 \%, P=0.103), 50 \mathrm{~ms}(\sim-15 \%$ and $-51 \%, P=0.038)$; RFDr: $30 \mathrm{msec}(\sim-22 \%$ and $-52 \%, P=0.044), 50 \mathrm{~ms}(\sim-11$ and $-54 \%$,

Table 2. Explosive force parameters from whole body vibration and sham groups at baseline (pre) and post training (post)

\begin{tabular}{|c|c|c|c|c|}
\hline \multirow{2}{*}{ Variable } & \multicolumn{2}{|c|}{ WBV $(n=7)$} & \multicolumn{2}{|c|}{ Sham $(n=7)$} \\
\hline & Pre & Post & Pre & Post \\
\hline Clmp at $30 \mathrm{msec}(\mathrm{N} . \mathrm{m} / \mathrm{sec})$ & $0.43 \pm 0.15$ & $0.35 \pm 0.15$ & $0.34 \pm 0.10$ & $0.19 \pm 0.10$ \\
\hline Clmp at $50 \mathrm{msec}(\mathrm{N} . \mathrm{m} / \mathrm{sec})$ & $1.33 \pm 0.47$ & $1.09 \pm 0.44$ & $1.07 \pm 0.31$ & $0.58 \pm 0.30$ \\
\hline Clmp at $100 \mathrm{msec}(\mathrm{N} \cdot \mathrm{m} / \mathrm{sec})^{\mathrm{a})}$ & $4.25 \pm 7.98$ & $4.16 \pm 4.32$ & $4.93 \pm 6.70$ & $1.07 \pm 3.05$ \\
\hline Clmp at $200 \mathrm{msec}(\mathrm{N} . \mathrm{m} / \mathrm{sec})^{\mathrm{a})}$ & $16.56 \pm 22.87$ & $16.07 \pm 13.56$ & $14.07 \pm 15.8$ & $3.23 \pm 8.46$ \\
\hline RFDr at $30 \mathrm{msec}(\% \mathrm{MVC} / \mathrm{sec})$ & $3.11 \pm 0.85$ & $2.31 \pm 0.68$ & $3.69 \pm 0.99$ & $1.73 \pm 0.73$ \\
\hline RFDr at $50 \mathrm{msec}(\% \mathrm{MVC} / \mathrm{sec})$ & $3.52 \pm 0.98$ & $2.78 \pm 0.72$ & $4.27 \pm 1.08$ & $1.97 \pm 0.81$ \\
\hline RFDr at 100 msec (\%MVC/sec $)^{\text {a) }}$ & $2.10 \pm 3.39$ & $2.53 \pm 1.90$ & $5.09 \pm 4.98$ & $0.97 \pm 2.69$ \\
\hline RFDr at $200 \mathrm{msec}(\% \mathrm{MVC} / \mathrm{sec})^{\mathrm{a})}$ & $2.22 \pm 1.84$ & $2.21 \pm 1.38$ & $2.99 \pm 2.92$ & $1.15 \pm 1.67$ \\
\hline
\end{tabular}

Values are presented as mean \pm standard error unless otherwise indicated.

CImp, contractile impulse; RFDr, relative rate of force development.

${ }^{a}$ Values presented as median \pm interquartile range. No significant difference between the groups before and after the interventions. 


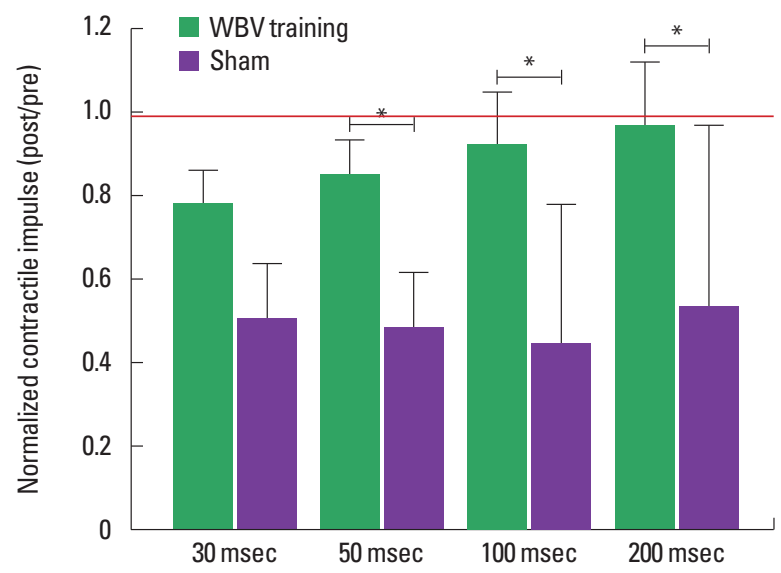

Fig. 2. Normalized contractile impulse (post/pre) from chronic kidney disease patients submitted to 3 months of whole body vibration (WBV) training or sham. * Significant difference between groups $(P<0.05)$. Red line indicates the reference value (i.e., the value where measure obtained post training period was equal to pre training). Data from normalized Clmp at 30 and $50 \mathrm{msec}$ are presented as mean \pm standard error, while data at 100 and $200 \mathrm{msec}$ are presented as median \pm interquartile range.

$P=0.008$ ) (for the WBV training and sham groups, respectively). For the parameters obtained at the late phase there was an lower decline for WBV group when compared to sham group: CImp: $100 \mathrm{msec}(\sim-8 \%$ and $-55 \%, P=0.025), 200 \mathrm{msec}(\sim-3 \%$ and $-46 \%, P=0.025)$; RFDr $100 \mathrm{msec}(\sim 0.01 \%$ and $-56 \%, P=0.033)$, $200 \mathrm{msec}(\sim-5 \%$ and $-36 \%, P=0.004)$ (for the WBV training and sham groups, respectively).

\section{DISCUSSION}

The present study aimed to evaluate whether WBV training may improve explosive force (Clam and RFDr) of the knee extensor muscles in CKD patients. Our main find indicated that 3 months of WBV training is potentially useful for to attenuate the explosive force reduction of these patients.

This positive effect of WBV training in CKD patients contradicts partially our hypothesis that there would be an increase in the parameters of explosive force. This apparently divergence may be explained by the clinical condition of the patients. In fact, about $50 \%$ of patients undergoing HD have lower limb myopathy (Arnold et al., 2016), which is strongly related to the risk of increased mortality in these patients (Matsuzawa et al., 2014).

Additionally, previous studies with elders observed improvements in the explosive strength and power of knee extensors after WBV when associated with other exercises (Bogaerts et al., 2007b; Tsuji et al., 2014), but it is possible that the WBV alone may avoid

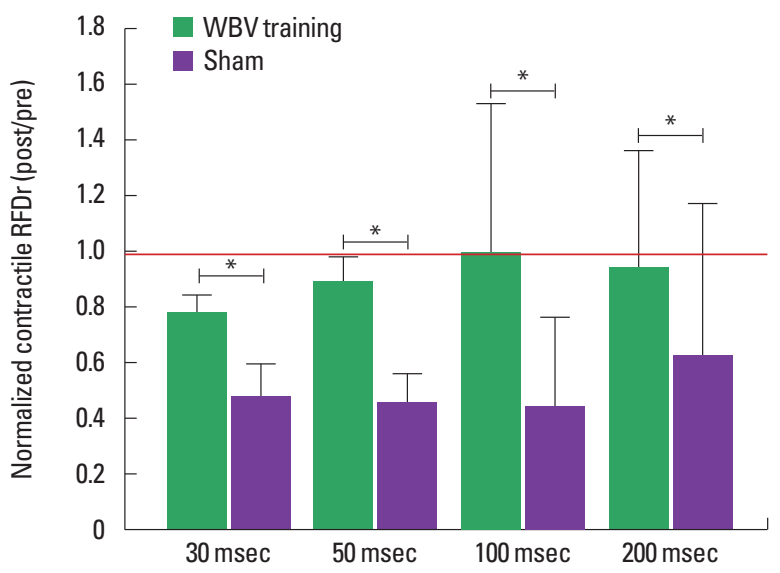

Fig. 3. Normalized relative rate of force development (RFDr) (POST/PRE) from chronic kidney disease patients submitted to three months of whole body vibration (WBV) training or sham. * Significant difference between groups $(P<0.05)$. Red line indicates the reference value (i.e., the value where measure obtained post training period was equal to pre training). Data from normalized RFDr at 30 and 50 msec are presented as mean \pm standard error, while data at 100 and 200 msec are presented as median \pm interquartile range.

the great explosive force decline associated to $\mathrm{CKD}$ and $\mathrm{HD}$, but could not be sufficient to induce significant increases in this variable. The results from Machado et al. (2010) may support this hypothesis, since they also observed that 10-week WBV training, for community-dwelling healthy elders, attenuated the decline but not provided significant muscle power improvements, while the control group experienced a significant decline of this variable.

The WBV is considered a safe and tolerable method to improve muscular performance (Bagheri et al., 2012; Cormie et al., 2006), however no study was found evaluating the effects of WBV on the explosive force of CDK patients. In fact, has been suggested (Buckinx et al., 2014; Rogan et al., 2015) that WBV can be applied to subjects unable to perform standard exercises, or to ones that the standard exercises are not suitable or well supported, owing to the physical weakness, as elders and CKD patients.

According to our results, previous studies showed no increase in muscle force gains after WBV training. In healthy elders, 6 weeks of WBV training did not increase maximal isokinetic strength of knee extensors and flexors (Perchthaler et al., 2015), and in stroke patients, 8 weeks of WBV training does not increases the maximum strength (Pang et al., 2013).

Although the explosive force has not increased in the present study, the decline attenuation generated by WBV training may be considered clinically useful outcome. The explosive force is important to prevent fall episodes in physically weakened populations, since postural reactions required to avoid falls after a sudden 
postural perturbation occur between 0-250 msec after the onset of muscle activity (Aagaard et al., 2002).

The explosive force depends on neural and muscular factors, so that it is usually analyzed/interpreted in two phases: (a) the early phase, comprising the first $0-50 \mathrm{msec}$, which depends more of neural factors; (b) the late phase, comprising the 100-200 msec, more associated to muscle properties (Aagaard et al., 2002; Maffiuletti et al., 2016). Based on this knowledge, in the present study, the attenuation in the explosive force reduction seem to be associated to both neural and muscular adaptations, since it were observed significant differences between WBV and SHAM groups in early and late phase of explosive force parameters.

The influence of WBV on neural factors may be explained by the activation of the TVR through changes in the sensitivity of the Ia afferents (Bagheri et al., 2012; Cardinale and Bosco, 2003), while muscular adaptations are probability associated to muscle cross-sectional area (Machado et al., 2010). Despite the low load involved in the WBV training, an attenuation of muscle hypotrophy in CDK patients could be plausible. In fact, it has been previously shown that 40 weeks of WBV without additional exercise promoted maintenance of the cross-sectional area in lower limbs of paraplegic patients (Masani et al., 2014). In addition, when associated with squatting exercises, 10 weeks of WBV may induce hypertrophy in older adults (Machado et al., 2010).

Data regarding the effects of WBV training in CKD patients are scarce. Recent pilot studies indicated that WBV training has the potential to attenuate bone deficits and improve physical function in this population (Doyle et al., 2017; Rajapakse et al., 2017). Our results may be of great clinical importance in the treatment of people with $\mathrm{CKD}$. Considering that this population has a high risk of falls, owing to the disease and HD treatment (Erken et al., 2016), attenuation of explosive force reduction may be useful to prevent such events. In fact, further studies should analyze the relationship between explosive force and clinical tests to evaluate falls risk (e.g., functional performance tests, balance tests, questionnaires, and others).

Recruit a homogeneous sample was a challenge, since CKD could be associated to many comorbidities and exhibit a wide range of clinical presentations. In addition, the long period of exercise training ( 3 months) leaded many patients to decline the invitation to engage in our study. Both factors limited the sample size and generated considerable discrepancies in baseline data (HD time and creatinine levels; see Table 1), which constitute limitations of our study. Further studies should consider an increase in the sample size and association of WBV training with other inter- ventions (e.g., kinesiotherapy, hydrotherapy, and others), since the WBV training was the unique intervention applied here. Analysis of muscle size and electromyographic activity also may improve the understanding of the mechanisms underlying explosive force parameters.

Three months of WBV may attenuate the explosive force reduction of the knee extensors in CKD patients. This intervention is therefore a potential tool for treatment programs aimed to improve the motor performance in this frail people, which could benefit the prevention of falls events, improving the quality of life of this population.

\section{CONFLICT OF INTEREST}

No potential conflict of interest relevant to this article was reported.

\section{ACKNOWLEDGMENTS}

This study was supported by grants from Fundação de Amparo à Ciência e Tecnologia do Estado de Pernambuco (FACEPE) APQ0250-4.08/13.

\section{REFERENCES}

Aagaard P, Simonsen EB, Andersen JL, Magnusson P, Dyhre-Poulsen P. Increased rate of force development and neural drive of human skeletal muscle following resistance training. J Appl Physiol (1985) 2002;93: 1318-1326.

Adams GR, Vaziri ND. Skeletal muscle dysfunction in chronic renal failure: effects of exercise. Am J Physiol Renal Physiol 2006;290:F753-761.

Arnold R, Issar T, Krishnan AV, Pussell BA. Neurological complications in chronic kidney disease. JRSM Cardiovasc Dis 2016;5:2048004016677 687.

Bagheri J, van den Berg-Emons RJ, Pel JJ, Horemans HL, Stam HJ. Acute effects of whole-body vibration on jump force and jump rate of force development: a comparative study of different devices. J Strength Cond Res 2012;26:691-696.

Bogaerts A, Delecluse C, Claessens AL, Coudyzer W, Boonen S, Verschueren SM. Impact of whole-body vibration training versus fitness training on muscle strength and muscle mass in older men: a 1-year randomized controlled trial. JGerontol A Biol Sci Med Sci 2007a;62: 630-635.

Bogaerts A, Verschueren S, Delecluse C, Claessens AL, Boonen S. Effects of whole body vibration training on postural control in older individ- 
uals: a 1 year randomized controlled trial. Gait Posture 2007b;26:309316.

Bogaerts AC, Delecluse C, Claessens AL, Troosters T, Boonen S, Verschueren SM. Effects of whole body vibration training on cardiorespiratory fitness and muscle strength in older individuals (a 1-year randomised controlled trial). Age Ageing 2009;38:448-454.

Borges LS, Fernandes MH, Schettino L, DA Silva Coqueiro R, Pereira R. Handgrip explosive force is correlated with mobility in the elderly women. Acta Bioeng Biomech 2015;17:145-149.

Braz Júnior DS, Dornelas de Andrade A, Teixeira AS, Cavalcanti CA, Morais $A B$, Marinho PE. Whole-body vibration improves functional capacity and quality of life in patients with severe chronic obstructive pulmonary disease (COPD): a pilot study. Int J Chron Obstruct Pulmon Dis 2015;10:125-132.

Buckinx F, Beaudart C, Maquet D, Demonceau M, Crielaard JM, Reginster JY, Bruyère O. Evaluation of the impact of 6-month training by whole body vibration on the risk of falls among nursing home residents, observed over a 12-month period: a single blind, randomized controlled trial. Aging Clin Exp Res 2014;26:369-376.

Cardinale M, Bosco C. The use of vibration as an exercise intervention. Exerc Sport Sci Rev 2003;31:3-7.

Cheema B, Abas H, Smith B, O'Sullivan A, Chan M, Patwardhan A, Kelly J, Gillin A, Pang G, Lloyd B, Singh MF. Progressive exercise for anabolism in kidney disease (PEAK): a randomized, controlled trial of resistance training during hemodialysis. J Am Soc Nephrol 2007;18:15941601.

Chen JL, Godfrey S, Ng TT, Moorthi R, Liangos O, Ruthazer R, Jaber BL, Levey AS, Castaneda-Sceppa C. Effect of intra-dialytic, low-intensity strength training on functional capacity in adult haemodialysis patients: a randomized pilot trial. Nephrol Dial Transplant 2010;25:19361943.

Cook WL, Tomlinson G, Donaldson M, Markowitz SN, Naglie G, Sobolev $\mathrm{B}$, Jassal SV. Falls and fall-related injuries in older dialysis patients. Clin J Am Soc Nephrol 2006;1:1197-1204.

Cormie P, Deane RS, Triplett NT, McBride JM. Acute effects of whole-body vibration on muscle activity, strength, and power. J Strength Cond Res 2006;20:257-261.

Doyle A, Chalmers K, Chinn DJ, McNeill F, Dall N, Grant CH. The utility of whole body vibration exercise in haemodialysis patients: a pilot study. Clin Kidney J 2017;10:822-829.

Erken E, Ozelsancak R, Sahin S, Yllmaz EE, Torun D, Leblebici B, Kuyucu YE, Sezer S. The effect of hemodialysis on balance measurements and risk of fall. Int Urol Nephrol 2016;48:1705-1711.

Greulich T, Nell C, Koepke J, Fechtel J, Franke M, Schmeck B, Haid D, Apelt S, Filipovic S, Kenn K, Janciauskiene S, Vogelmeier C, Koczulla
AR. Benefits of whole body vibration training in patients hospitalised for COPD exacerbations - a randomized clinical trial. BMC Pulm Med 2014;14:60.

Haas CT, Turbanski S, Kessler K, Schmidtbleicher D. The effects of random whole-body-vibration on motor symptoms in Parkinson's disease. NeuroRehabilitation 2006;21:29-36.

Kopple JD, Storer T, Casburi R. Impaired exercise capacity and exercise training in maintenance hemodialysis patients. J Ren Nutr 2005;15:4448.

Lam FM, Lau RW, Chung RC, Pang MY. The effect of whole body vibration on balance, mobility and falls in older adults: a systematic review and meta-analysis. Maturitas 2012;72:206-213.

Machado A, García-López D, González-Gallego J, Garatachea N. Wholebody vibration training increases muscle strength and mass in older women: a randomized-controlled trial. Scand J Med Sci Sports 2010; 20:200-207.

Maffiuletti NA, Aagaard P, Blazevich AJ, Folland J, Tillin N, Duchateau J. Rate of force development: physiological and methodological considerations. Eur J Appl Physiol 2016;116:1091-1116.

Masani K, Alizadeh-Meghrazi M, Sayenko DG, Zariffa J, Moore C, Giangregorio L, Popovic MR, Catharine Craven B. Muscle activity, crosssectional area, and density following passive standing and whole body vibration: a case series. J Spinal Cord Med 2014;37:575-581.

Matsuzawa R, Matsunaga A, Wang G, Yamamoto S, Kutsuna T, Ishii A, Abe Y, Yoneki K, Yoshida A, Takahira N. Relationship between lower extremity muscle strength and all-cause mortality in Japanese patients undergoing dialysis. Phys Ther 2014;94:947-956.

Oliveira FB, Oliveira AS, Rizatto GF, Denadai BS. Resistance training for explosive and maximal strength: effects on early and late rate of force development. J Sports Sci Med 2013;12:402-408.

Pang MY, Lau RW, Yip SP. The effects of whole-body vibration therapy on bone turnover, muscle strength, motor function, and spasticity in chronic stroke: a randomized controlled trial. Eur J Phys Rehabil Med 2013;49:439-450.

Perchthaler D, Grau S, Hein T. Evaluation of a six-week whole-body vibration intervention on neuromuscular performance in older adults. J Strength Cond Res 2015;29:86-95.

Pereira CC, Neves FD. Conforto humano e limites de percepção para vibrações verticais. Rem Rev Esc Minas 2006;59:271-278.

Rajapakse CS, Leonard MB, Kobe EA, Slinger MA, Borges KA, Billig E, Rubin CT, Wehrli FW. The efficacy of low-intensity vibration to improve bone health in patients with end-stage renal disease is highly dependent on compliance and muscle response. Acad Radiol 2017;24: 1332-1342.

Rietschel E, van Koningsbruggen S, Fricke O, Semler O, Schoenau E. Whole 
body vibration: a new therapeutic approach to improve muscle function in cystic fibrosis? Int J Rehabil Res 2008;31:253-256.

Rittweger J. Vibration as an exercise modality: how it may work, and what its potential might be. Eur J Appl Physiol 2010;108:877-904.

Rogan S, de Bruin ED, Radlinger L, Joehr C, Wyss C, Stuck NJ, Bruelhart Y, de Bie RA, Hilfiker R. Effects of whole-body vibration on proxies of muscle strength in old adults: a systematic review and meta-analysis on the role of physical capacity level. Eur Rev Aging Phys Act 2015; 12:12.

Schettino L, Luz CP, de Oliveira LE, de Assunção PL, da Silva Coqueiro R, Fernandes MH, Brown LE, Machado M, Pereira R. Comparison of explosive force between young and elderly women: evidence of an earlier decline from explosive force. Age (Dordr) 2014;36:893-898.

Schuhfried O, Mittermaier C, Jovanovic T, Pieber K, Paternostro-Sluga T. Effects of whole-body vibration in patients with multiple sclerosis: a pilot study. Clin Rehabil 2005;19:834-842.

Skelton DA, Kennedy J, Rutherford OM. Explosive power and asymmetry in leg muscle function in frequent fallers and non-fallers aged over 65. Age Ageing 2002;31:119-125.

Storer TW, Casaburi R, Sawelson S, Kopple JD. Endurance exercise train- ing during haemodialysis improves strength, power, fatigability and physical performance in maintenance haemodialysis patients. Nephrol Dial Transplant 2005;20:1429-1437.

Sundstrup E, Jakobsen MD, Andersen LL, Andersen TR, Randers MB, Helge JW, Suetta C, Schmidt JF, Bangsbo J, Krustrup P, Aagaard P. Positive effects of 1 -year football and strength training on mechanical muscle function and functional capacity in elderly men. Eur J Appl Physiol 2016;116:1127-1138.

Tsuji T, Yoon J, Aiba T, Kanamori A, Okura T, Tanaka K. Effects of wholebody vibration exercise on muscular strength and power, functional mobility and self-reported knee function in middle-aged and older Japanese women with knee pain. Knee 2014;21:1088-1095.

Wang E, Nyberg SK, Hoff J, Zhao J, Leivseth G, Tørhaug T, Husby OS, Helgerud J, Richardson RS. Impact of maximal strength training on work efficiency and muscle fiber type in the elderly: implications for physical function and fall prevention. Exp Gerontol 2017;91:64-71.

Yang F, King GA, Dillon L, Su X. Controlled whole-body vibration training reduces risk of falls among community-dwelling older adults. J Biomech 2015;48:3206-3212. 